Ann. Sci. forest., 1973, 30 (2), 175-190.

\title{
ÉVOLUTION DES ÉLÉMENTS MINÉRAUX DISSOUS \\ DANS LES PRÉCIPITATIONS ET LES PERCOLATS DU SOL A TRAVERS UN ÉCOSYSTÈME FORESTIER*
}

\author{
M. RAPP et D.-W. COLE \\ C.N.R.S., C.E.P.E.L.E., B.P. 5051, 34033 Montpellier Cedex \\ University of Washington, College of Forest Resources, \\ Seattle, 98105, Washington (U.S.A.)
}

\section{RÉSUMÉ}

L'évolution de la composition en $\mathrm{Na}, \mathrm{K}, \mathrm{Ca}, \mathrm{Mg}$ des précipitations s'infiltrant dans le sol a été étudiée dans une forêt de Douglas de la côte Nord-Ouest des États-Unis au moyen de lysimètres à succion.

Le bilan de ces quatres éléments, circulant sous forme dissoute, a été établi pour l'écosystème entier et pour le sol en particulier.

Les variations de la concentration des percolats à différentes profondeurs ont été analysées et des explications de la mise en solution et du transport des cations dans le sol sont avancées.

Un contrôle par irrigation du sol en place au moyen d'une solution déminéralisée confirme les hypothèses émises sur les mécanismes de la cinétique des bioéléments dans le sol.

\section{INTRODUCTION}

Si de nombreux travaux concernant le cycle biogéochimique des éléments minéraux dans les écosystèmes forestiers ont vu le jour récemment, notamment dans le cadre du P.B.I., peu ont trait aux quantités et à la nature des éléments évoluant à travers le sol, et aux mécanismes impliqués dans cette circulation. Or, c'est là un problème essentiel du fonctionnement des écosystèmes. Il intervient à la fois dans la disponibilité des bioéléments pour la végétation, dans l'évolution des sols, ainsi que dans la rétention et le devenir dans le milieu édaphique des polluants minéraux éventuellement contenus dans les précipitations.

Nous avons étudié certains aspects de ce problème dans une forêt de Douglas (Pseudotsuga Menziesii Franco), sur sol podzolique et sous climat océanique du nord-ouest des États-Unis. Un certain nombre d'études sur le même sujet ont déjà été réalisées au même endroit par Gessel et Cole (1965), Mc Coll et Cole (1968), Cole et Ballard (1968). Le

\footnotetext{
* Ce travail a été réalisé dans le cadre de la convention d'échange C.N.R.S. (France) - N.S.F. (U.S.A.).
} 
présent travail porte sur l'évolution de la composition chimique des eaux de pluie à travers le couvert végétal dans le but d'établir à la fois un bilan de la circulation des éléments majeurs à différents niveaux de l'écosystème et de déterminer les mécanismes responsables de ce flux dans le milieu édaphique. Les observations faites en condition naturelle ont à cet effet été contrôlées et vérifiées par une expérience d'irrigation.

\section{LA STATION}

La station expérimentale A. ThOmSOn à Cedar River a été décrite en détail par CoLE et Gessel (1968).

Rappelons que c'est un peuplement équien de Douglas, plantés en 1931. Actuellement les arbres ont une hauteur moyenne de 18 mètres, une densité de 2200 pieds à l'hectare et une surface terrière de $37 \mathrm{~m}^{2} /$ ha.

La strate herbacée, dont le recouvrement est de $100 \%$, est constituée essentiellement de :

Gaultheria shallon Pursh.

Berberis nervosa Pursh.

Pteridium aquilinum $\mathrm{L}$.

Vaccinium parvifolium Smith

Linnaea borealis L., var. americana Forbes

ainsi que d'un important tapis de mousses.

Le sol est de type podzolique (Haplorthod typique), sur matériau glaciaire à granulométrie grossière. Cole, Gessel et Dice (1967) en donnent la description suivante :

$2-0 \mathrm{~cm}$ Litière partiellement décomposée.

$0-2 \mathrm{~cm}$ Couleur brun foncé (10 YR); texture sablo-limoneuse, structure particulaire friable; très perméable; quelques concrétions; passage irrégulier avec l'horizon suivant; beaucoup de racines.

2 - 7 Couleur brun jaune foncé (10 YR 4/4); texture sablo-limoneuse; structure particulaire friable; meuble; passage irrégulier avec l'horizon suivant; beaucoup de racines.

7 - 30 Couleur jaune brun (10 YR 5/4); texture limono-sableuse; structure particulaire; meuble; passage irrégulier mais net avec l'horizon inférieur; présence de racines.

$>30$ Couleur jaune brun (10 YR 5/4); texture sableuse (sable grossier et gravillons); structure particulaire; meuble; peu de racines.

Les caractéristiques chimiques sont résumées sur le tableau 1.

Le climat est doux, de type océanique, typique des élévations moyennes de la côte nordouest des États-Unis. La température moyenne du mois le plus froid (janvier) est de $1,7^{\circ} \mathrm{C}$., celle du mois le plus chaud (juillet) de $16,7^{\circ} \mathrm{C}$. Les précipitations annuelles s'élèvent en moyenne à $1360 \mathrm{~mm}$, tombant essentiellement sous forme de pluies d'automne ou de printemps (70\% d'octobre à mars). 
TABLEAU 1

Caractéristiques chimiques du sol de la placette expérimentale Chemical characteristics of the Cedar River soil

\begin{tabular}{|c|c|c|c|c|c|c|c|c|}
\hline \multirow{3}{*}{$\begin{array}{l}\text { Niveau } \\
\mathrm{cm}\end{array}$} & \multirow{3}{*}{$\mathrm{pH}$} & \multicolumn{4}{|c|}{ méq / $100 \mathrm{~g}$} & \multirow{3}{*}{$\begin{array}{c}\text { Taux } \\
\text { de } \\
\text { saturation }\end{array}$} & \multirow{3}{*}{$\begin{array}{l}\mathrm{N} \\
\% \\
\%\end{array}$} & \multirow{3}{*}{$\begin{array}{c}\text { Matière } \\
\text { organique } \\
\%\end{array}$} \\
\hline & & \multirow{2}{*}{$\begin{array}{l}\text { Capacité } \\
\text { d'échange }\end{array}$} & \multicolumn{3}{|c|}{$\begin{array}{c}\text { Cations } \\
\text { échangeables }\end{array}$} & & & \\
\hline & & & K & $\mathrm{Ca}$ & $\mathrm{Mg}$ & & & \\
\hline $0-2,5$ & 5,4 & 10,83 & 0,25 & 1,88 & 2,10 & 38,8 & 0,15 & 8,25 \\
\hline $2,5-5-30$ & $\begin{array}{l}5,5 \\
5,6\end{array}$ & 4,07 & 0,08 & 0,25 & 0,09 & 10,6 & 0,025 & $\begin{array}{l}2,54 \\
1,86\end{array}$ \\
\hline $30-60$ & 5,85 & 1,10 & 0,002 & 0,13 & 0,08 & 19,8 & 0,004 & 0,30 \\
\hline
\end{tabular}

\section{MÉTHODES D'ÉTUDES}

\section{Récolte des échantillons}

Les pluies ont été récoltées après chaque précipitation ou période pluvieuse au cours des mois de mars, avril et mai 1971, grâce à trois pluviomètres. L'un était disposé dans une clairière (pluie incidente), deux autres sous les arbres (égouttement). De plus, les eaux d'écoulement ont été prélevées à la base des troncs de deux arbres.

Les eaux de percolation ont été captées par des lysimètres à succion, selon le principe décrit par Cole (1968). Ces lysimètres sont constitués d'un disque en alundum, dont la face inférieure est recouverte et fermée hermétiquement par une plaque en matière plastique. Lorsque le système est saturé d'eau, une légère dépression $(0,1$ atmosphère) appliquée dans l'interstice entre le disque poreux et la plaque en plastique permet d'aspirer l'eau du sol vers l'intérieur du lysimètre et de là vers un récipient collecteur.

Les différents appareils étaient disposés sous la litière $(\mathrm{F})$, sous l'horizon $\mathrm{A}(-7 \mathrm{~cm})$, dans et sous l'horizon $\mathrm{B}$, respectivement à $-15(\mathrm{~B})$ et $-30 \mathrm{~cm}\left(\mathrm{~B}^{\prime}\right)$ et dans l'horizon $\mathrm{C}(-100 \mathrm{~cm})$.

Trois répétitions ont été réalisées pour les deux premiers niveaux ( $\mathrm{F}$ et $\mathrm{A}$ ) avec des lysimètres qui étaient en place depuis plusieurs années, deux pour les trois autres, installés au début de notre expérience.

\section{Arrosage du profil}

Après une période sèche, la surface du sol recouvrant un profil complet de lysimètres a été arrosée durant deux heures avec un volume d'eau correspondant à $20 \mathrm{~mm}$ de pluie. Des échantillons de volume identique $(100 \mathrm{ml})$ ont été récoltés au long d'une période de dix heures suivant l'arrosage. Un dernier prélèvement a été effectué au bout de 36 heures.

\section{Analyses chimiques}

Elles ont consisté à déterminer le $\mathrm{pH}$, les bicarbonates, obtenus par titration à l'acide sulfurique $\mathrm{N} / 50$ à pH 4,5 (RAinwater et Thatcher, 1960) et les quatre cations majeurs Na, K, Ca, Mg. Ces derniers ont été dosés directement par spectrophotométrie à absorption atomique. 


\section{BILAN DE L'EAU TRAVERSANT L'ÉCOSYSTÈME}

Nous avons indiqué sur la figure 1 les hauteurs d'eau moyennes, mesurées durant 18 périodes pluvieuses, s'étalant de mars à mai 1971, à différents niveaux de l'écosystème.

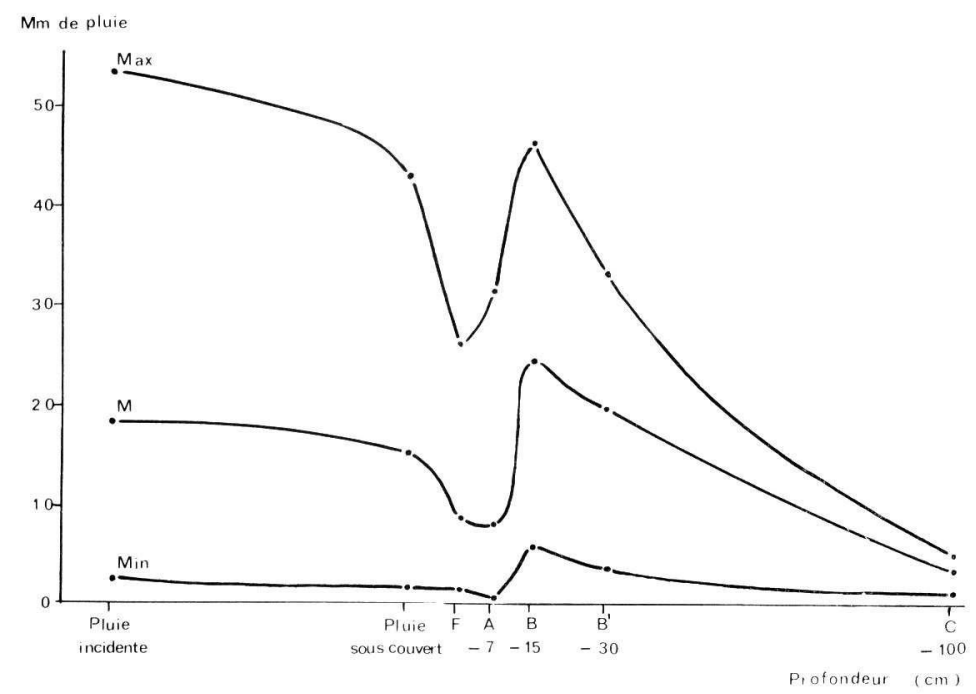

FIG. 1. - Évolution du flux d'eau à travers l'écosystème Max. - Min. : hauteurs d'eau maximales et minimales observées pendant une période pluvieuse M : hauteur moyenne du flux d'eau pour la période d'observation (mars, avril, mai 1971)

FIG. 1. - Evolution of the water flow through the ecosystem

Max. - Min. : maximum and minimum water levels observed during a storm, M mean value of the water flow for the measurement period (March, April, Mai 1971)

L’interception par le couvert végétal est de l'ordre de $30 \%$. L'égouttement représente de 75 à $80 \%$ de la pluie arrivant au sol, l'écoulement le long des troncs le restant.

Dans les cinq niveaux du sol, l'on assiste à une diminution régulière de la quantité d'eau percolant de la surface, jusqu'à un mètre de profondeur, sauf pour les niveaux B et $\mathbf{B}^{\prime}$. A ces niveaux, nous avons toujours recueilli des volumes largement supérieurs à ceux captés audessus ou en dessous. Plusieurs hypothèses peuvent être avancées pour expliquer ces variations :

Certaines concernent plus particulièrement des problèmes techniques. Des circulations préférentielles peuvent apparaître le long des zones où le sol a été perturbé au moment de l'installation des plaques lysimétriques; avec le temps, ce phénomène s'atténue et le sol se restructure. Le fait d'appliquer aux lysimètres une dépression de 0,1 atmosphère, entraîne lui, surtout en période humide, une succion plus importante d'eau. Le volume de sol dans lequel l'eau est collectée n'est plus dans ce cas un cylindre dont la base est la plaque lysimétrique, mais un tronc de cône renversé dont la plaque serait la petite base. 
D'autres explications sont liées à l'hétérogénéité du sol : ainsi, il peut présenter des zones plus hydromorphes, ou à capacité de rétention en eau plus élevée. Ceci se répercute sur les quantités recueillies par les lysimètres implantés à ces endroits. De même, la texture du sol peut influer sur le contact avec la plaque poreuse et favoriser plus ou moins le passage de la solution d'un milieu à l'autre.

Dans le cas présent, les deux types d'explications sont valables. Les fortes quantités d'eau récoltées à $-15 \mathrm{~cm}$ proviennent essentiellement d'une infiltration rapide, le long de zones de perturbation provoquées par la mise en place des lysimètres au début de l'expérimentation. Nous en avons eu une vérification au moment d'un premier essai d'irrigation, où nous avons assisté à un flux instantané de l'eau d'arrosage vers le lysimètre à $-15 \mathrm{~cm}$. Un dispositif expérimental particulier a permis de corriger cet inconvénient lors des essais ultérieurs (Tabl. 2). Mais il n'était pas utilisable en condition naturelle.

Pour le niveau $-30 \mathrm{~cm}$, nous assistons certainement à une conjonction entre la présence d'une zone à capacité de rétention plus élevée et l'existence d'une succion supérieure à la normale, qui y dispose d'un plus grand volume d'eau. L'hypothèse de zones d'infiltration rapide, consécutives à l'installation des appareils, peut être éliminée à cette profondeur où nous avons toujours recueilli les premiers $100 \mathrm{ml}$ d'eau entre 90 et 100 minutes après le début de l'arrosage.

Enfin, il ne faut pas oublier le rôle des végétaux qui consomment des grandes quantités d'eau dans la zone racinaire, donc essentiellement dans les 40 premiers centimètres. Cette utilisation peut être importante à partir d'avril et de mai, avec le démarrage de la période de végétation active.

TABLEAU 2

Volumes d'eau recueillis par un arrosage et après deux périodes pluvieuses,

* sans dispositif de correction

Volume of water recovered after irrigation and after two storms,

* without correction device

\begin{tabular}{c|c|c|c}
\hline \hline Niveau & Irrigation & $03 / 24 / 71$ & $03 / 31 / 71$ \\
\hline F & 959 & 987 & 1067 \\
\hline A & 1091 & 917 & 963 \\
\hline B & 957 & $2270 *$ & $3090 *$ \\
\hline B' & 1826 & 1625 & 1890 \\
\hline C & 225 & 280 & 320 \\
\hline \hline
\end{tabular}

Le flux d'eau à travers le profil varie aussi durant la période de précipitation ou d'irrigation et montre un décalage régulier depuis la surface jusqu'à $30 \mathrm{~cm}$ de profondeur. $\mathrm{A}$ un 
mètre, il disparaît et nous n'avons toujours récolté qu'un seul échantillon après les expériences d'irrigation.

Cette étude physique de l'évolution des eaux de percolation à travers le substrat édaphique après la pluie a surtout mis en évidence la nécessité d'appliquer un facteur de correction aux résultats de l'analyse chimique des niveaux $\mathbf{B}$ et $\mathbf{B}^{\prime}$. Pour cela, nous avons admis que les volumes d'eau drainés à un niveau quelconque ne pouvaient en aucun cas être supérieurs à ceux trouvés en $\mathrm{F}$ et nous avons calculé les quantités d'éléments de la solution du sol à -15 et - $30 \mathrm{~cm}$ d'après les volumes d'eau mesurés sous la litière.

Ceci peut entraîner une certaine erreur sur les valeurs absolues du fait que la concentration d'une solution peut varier en fonction du volume. Mais dans un site très voisin, Bourgeois et LAVKulich (1972) n'ont observé aucun changement dans la concentration cationique de percolats recueillis au même moment à deux endroits différents, alors que les volumes variaient fortement. Ceci confirme nos propres constatations : les fluctuations en concentration de solutions de volume différent, durant des périodes rapprochées, sont négligeables par rapport à celles résultant de l'évolution de l'activité biologique du sol avec le temps.

\section{L'ÉVOLUTION DES ÉLÉMENTS MINÉRAUX}

A TRAVERS LA PHYTOCÉNOSE ET LE SOL

La circulation des éléments dissous dans les eaux de pluie et les eaux de drainage consécutives aux précipitations peut être envisagée sous différentes formes. Nous allons successivement passer en revue le bilan pondéral dans l'écosystème entier et au niveau du sol en particulier; puis, l'évolution des éléments majeurs à travers le sol en condition naturelle, à la fois du point de vue quantitatif et de la relation anions-cations. Enfin, nous étudierons les variations de la composition chimique des eaux de drainage durant un cycle d'infiltration.

\section{Le bilan des éléments minéraux mis en circulation par les eaux de pluie à travers l'écosystème}

Nous avons établi ce bilan à deux niveaux. Le premier concerne l'écosystème global et représente la différence entre les apports par les eaux météoriques et les sorties par drainage vers le substrat géologique. Le second porte uniquement sur le sol de l'écosystème. Il représente la différence entre l'entrée d'éléments dans le sol après pluviolessivage de la frondaison et les exportations vers le substrat ou la phytocénose.

Dans les deux cas il faut remarquer que les quantités d'éléments regroupées sous le terme " entrée » ne correspondent pas exactement à celles de la «sortie ». Ces dernières représentent à la fois la somme des éléments dissous dans les eaux de pluie et ceux lessivés à partir de la litière en décomposition ou entraînés à partir du sol traversé.

A la vue des résultats consignés sur le tableau 3, l'on constate que le bilan est positif pour les deux monovalents, négatif pour le magnésium. Pour le calcium, il est négatif au niveau de l'écosystème entier, positif pour son sol. Ceci signifie que les précipitations sont, exception faite du magnésium, responsables d'un plus grand apport d'éléments biogènes au 
TABLEAU 3

Composition chimique et bilan des précipitations traversant l'écosystème durant les mois de mars, avril et mai 1971 ( $\mathrm{g} / \mathrm{m}^{2} / 3$ mois)

Chemical composition and its variation for the precipitations passing through the ecosystem during the month March, April and Mai 1971 (g/m²/3 months)

\begin{tabular}{|c|c|c|c|c|}
\hline & K & $\mathrm{Na}$ & $\mathrm{Ca}$ & $\mathrm{Mg}$ \\
\hline Apport de l'extérieur (1). & 0,15 & 0,50 & 0,13 & 0,03 \\
\hline Apport au sol (2).. & 0,46 & 0,60 & 0,26 & 0,07 \\
\hline Lessivé à 1 mètre (3) & 0,02 & 0,06 & 0,22 & 0,19 \\
\hline Bilan pour l'écosystème 1-3 & $+0,13$ & $+0,44$ & $-0,09$ & $-0,16$ \\
\hline Bilan pour le sol 2-3 & $+0,44$ & $+0,54$ & $+0,04$ & $-0,12$ \\
\hline
\end{tabular}

sol, que d'un départ hors de l'écosystème. Bien entendu, le bilan du sol n'est que partiel, puisqu'il ne concerne, ni l'absorption d'éléments minéraux par la végétation à partir du sol, ni leur restitution sous forme de litière. Ces derniers phénomènes peuvent masquer, voire changer le bilan réel du milieu édaphique, donc celui du « turnover » intrinsèque à l'écosystème.

\section{La cinétique des éléments minéraux à travers le sol}

Nous avons représenté sur la figure 2 les quantités totales d'éléments minéraux mises en circulation aux différents niveaux analysés. Deux faits apparaissent. L'un concerne le rôle de la strate herbacée et de la litière dans l'évolution du flux d'éléments à travers le profil, l'autre les différences entre les quatre éléments étudiés.

L'on assiste à une diminution considérable de la quantité d'éléments dissous, quand les eaux de pluie traversent la couche L et F. Elle est plus importante pour les alcalins que pour les alcalino-terreux. Alors que les eaux de pluviolessivage sont très riches en sodium, potassium et à un degré moindre en calcium, les percolats ayant traversé la couche $\mathrm{L}$ et $\mathrm{F}$ le sont beaucoup moins. Or, à première vue, l'on s'attendrait au contraire. Les lessivats devraient être plus concentrés par suite de la décomposition de la litière et de la libération concomitante d'éléments minéraux.

On constate par ailleurs une différence dans l'évolution individuelle des quatre éléments à travers le profil. Le potassium, après avoir augmenté considérablement à la suite du pluviolessivage, diminue graduellement lors de l'infiltration des précipitations, pour être entraîné en quantités infimes à un mètre de profondeur. Pour les trois autres éléments, l'enrichissement est beaucoup moins important lors de la traversée du couvert forestier. Dans l'horizon A il y a une certaine stabilité dans la teneur en sodium et en calcium, alors que celle en magnésium 
augmente. Au contraire, entre 7 et $30 \mathrm{~cm}$, les lessivats s'enrichissent aussi bien en calcium, qu'en magnésium ou en sodium. Enfin, dans les 70 derniers centimètres, l'on assiste à une légère fixation des éléments entraînés à partir des 30 premiers centimètres du profil.

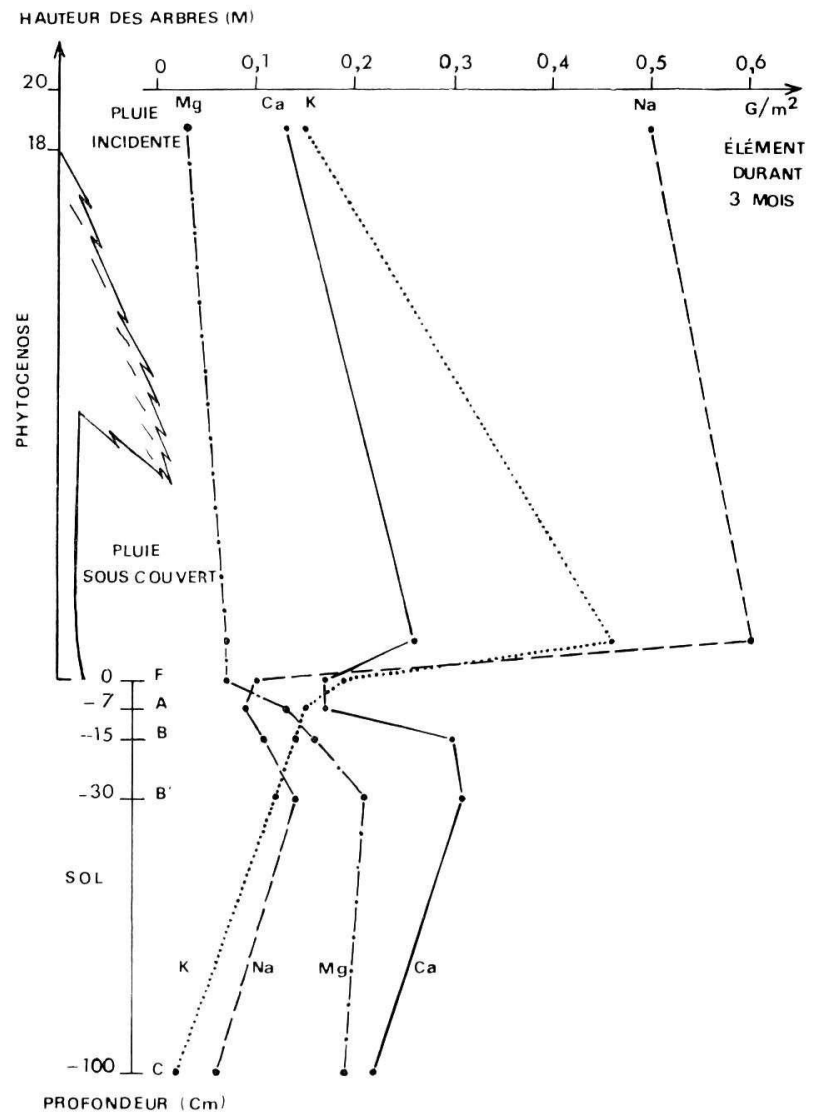

FIG. 2. - Évolution quantitative des cations majeurs à travers le peuplement étudié durant les trois mois d'observations $\left(\mathrm{g} / \mathrm{m}^{2} / 3\right.$ mois)

FIG. 2. - Evolution of the amount of $\mathrm{Na}, \mathrm{K}, \mathrm{Ca}, \mathrm{Mg}$ through the site during the three months of study $\left(\mathrm{g} / \mathrm{m}^{2} / 3\right.$ months $)$

La figure 3 représente un autre mode d'expression de l'évolution des éléments majeurs à travers le profil. Elle indique l'enrichissement en éléments minéraux de la solution du sol, non plus d'une manière pondérale, mais l'un par rapport à l'autre. Pour cela, nous avons établi la relation de la teneur en éléments minéraux de la solution du sol récoltée aux niveaux $\mathrm{A}, \mathrm{B}$, $\mathrm{B}^{\prime}$ et $\mathrm{C}$ par rapport à celle recueillie à la base de la couche $\mathrm{F}$.

Globalement les faits précédents sont confirmés : diminution graduelle de la teneur en potassium au fur et à mesure que la pluie pénètre plus profondément dans le sol, accroisse- 
ment des quantités entraînées depuis $F$ jusqu'à $-30 \mathrm{~cm}$ pour les trois autres éléments, suivi d'une diminution dans les $70 \mathrm{~cm}$ suivants. Mais si pondéralement on avait la succession :

$$
\mathrm{Ca}>\mathrm{Mg}>\mathrm{Na},
$$

elle devient dans ce second mode d'expression :

$$
\mathrm{Mg}>\mathrm{Ca}>\mathrm{Na} .
$$

Dans l'écosystème étudié, le magnésium est l'élément majeur entraîné le plus intensément lors du lessivage du sol par les précipitations. KINDERIs (1970) l'a également constaté sous le sol cultivé. De même, il a montré que sur l'ensemble d'une année, c'est au printemps que ce lessivage est surtout important.

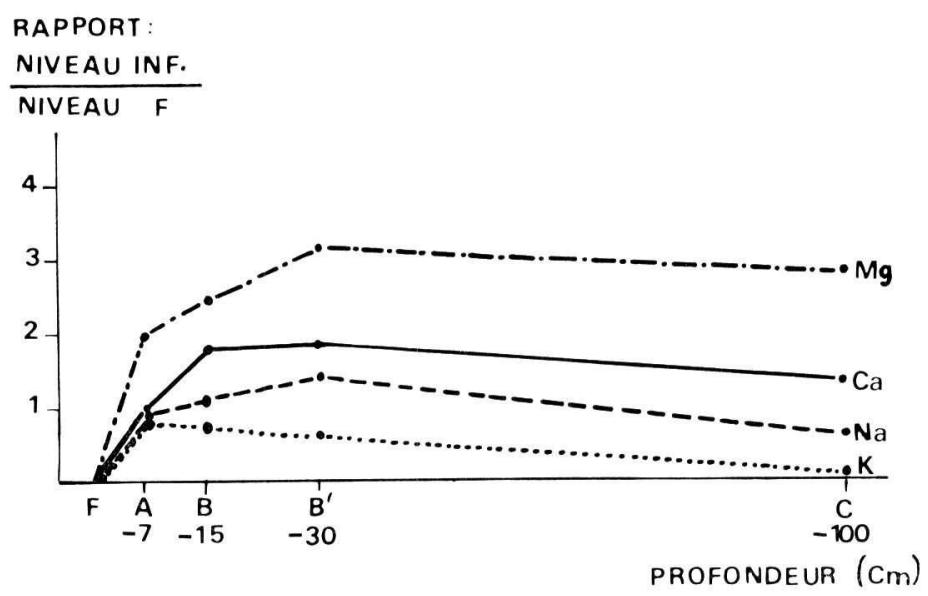

FIG. 3. - Facteur d'augmentation du lessivage des éléments majeurs en fonction de la profondeur du profil. (exprimé par rapport aux quantités totales et corrigé pour les niveaux $-15 \mathrm{et}-30 \mathrm{~cm}$ )

FIG. 3. - Coefficient of increase of mineral elements leached, plotted against the depth of the soil (as a percent of the total amount and corrected for the -15 and $-30 \mathrm{~cm}$ levels)

Ceci se répercute aussi sur la composition du complexe absorbant. A $-30 \mathrm{~cm}$, le potassium et le calcium fixés sur le complexe organo-minéral représentent encore $1 / 4$ et $1 / 8$ de celui présent en $\mathrm{F}$, alors que le magnésium, dont le lessivage est proportionnellement plus important, ne constitue plus que $1 / 25$.

Plusieurs faits peuvent être avancés pour expliquer l'évolution et le chimisme des éléments minéraux à travers le sol. On peut les regrouper en trois grandes catégories :

- Activité biologique de la phytocénose, de la litière et des microorganismes du sol.

- Disponibilité des éléments fixés sur le complexe absorbant ou en réserve dans la trame minérale. Phénomènes d'échange solution-complexe.

- Relations anions-cations dans le sol. 
L'importante fixation d'éléments biogènes lors du contact des précipitations avec le sol peut résulter en grande partie de l'activité du système biologique constitué par le tapis de mousses. Kilbertus (1970) a indiqué que les mousses étaient susceptibles de retenir 14 fois leur poids sec d'eau, ce qui correspond à une valeur se situant entre $1,5 \mathrm{et} 2 \mathrm{~mm}$ de pluie. Ce rôle des mousses doit être très important dans le cas du potassium et du sodium. Ce dernier, en quantité importante dans les précipitations, semble être immobilisé dans l'horizon F. Au contraire, des observations effectuées sur un sol forestier dépourvu de bryophytes, situé aux bords de la Méditerranée, indiquent que tout le sodium apporté par les eaux météoriques percole vers le substrat géologique (RAPP, 1971). De plus, l'horizon humifère conditionne lui aussi l'évolution de la solution du sol. Juste (1965) a distingué deux types d'évolution, dénommés «mobilisation biologique » et « mobilisation chimique ». La première est caractérisée par une accumulation des éléments dans les horizons supérieurs du profil, un appauvrissement des lessivats. Elle est essentiellement fonction des propriétés de l'horizon organique et concerne le potassium et le sodium.

Dans le cas présent, la fixation importante d'alcalins dans l'horizon de surface semble résulter à la fois de l'activité biologique des mousses et des propriétés d'insolubilisation biologique (Duchaufour, 1968). Les alcalino-terreux au contraire sont mobilisés par des phénomènes physico-chimiques. Néanmoins il est possible qu'une partie du calcium puisse aussi être immobilisée par les mousses.

Dans le sol, l'évolution du potassium est due essentiellement à sa très grande mobilité dans les systèmes biologiques. Cet alcalin est utilisé très promptement par les racines, pour être remis non moins rapidement en circulation, par récrétion foliaire et pluviolessivage.

Pour les autres cations au contraire, l'absorption par les racines, qui se situe dans les 40 premiers centimètres, est certainement beaucoup moins importante, surtout au début de notre période d'observation, à la fin de l'hiver. Mais cette absence de dérivation vers la phytocénose n'explique pas à elle seule l'accroissement de la concentration de la solution du sol avec la profondeur traversée, phénomène qui correspond à la «mobilisation chimique » de JUSTE (1965).

Les causes en sont variées. Il y a certainement un équilibre entre la quantité d'ions en solution et la quantité totale disponible sur le complexe. Cette dernière a été estimée à $75 \mathrm{~g} / \mathrm{m}^{2}$ de calcium et $23 \mathrm{~g} / \mathrm{m}^{2}$ de potassium (COLE, Gessel, Dice, 1967) pour l'ensemble du profil. Mais la répartition n'est pas uniforme, comme nous l'avons indiqué sur le tableau 1, et l'équilibre est susceptible d'être déplacé. Ainsi, au-delà des trente premiers centimètres, l'on assiste à une fixation partielle sur le complexe, qui résulte certainement d'un changement dans l'équilibre entre la concentration de la solution du sol et celle du complexe absorbant. Changement qui résulte lui-même de l'enrichissement du percolat dans les horizons supérieurs.

Une grande importance doit être accordée aux anions bicarboniques comme agents responsables de la solubilisation et du transport des cations (Shilova et KorovkinA, 1965; MC Coll et Cole, 1968). Ainsi, le calcium et le magnésium migreraient surtout sous forme de bicarbonates, qui trouveraient eux-mêmes leur origine dans le $\mathrm{CO}_{2}$ résultant de la décomposition de la litière, de l'humus, et surtout de la respiration racinaire. A l'acide carbonique s'ajoutent des acides et des substances organiques, qui peuvent, pour les uns, donner lieu à des échanges d'ions $\mathrm{H}^{+}$et de cations métalliques, pour les autres, constituer des complexes organo-minéraux simples. Sur la figure 4 nous avons indiqué la teneur, exprimée en milliéquivalents, de la solution du sol en $\mathrm{K}, \mathrm{Ca}, \mathrm{Mg}$ et $\mathrm{Na}$ et en bicarbonates. Cette dernière valeur 
représente en réalité, non seulement la totalité des bicarbonates, mais encore certains sels d'acides organiques faibles, dont les constantes d'acidité sont voisines du pH 4,5. L'on constate que les bicarbonates et les sels d'acides organiques augmentent, tout comme les cations métalliques, dans les 30 premiers centimètres. Puis, la teneur reste stable pour les bicarbonates, alors que les cations en solution diminuent. De ce fait, à un mètre de profondeur, l'on peut admettre que les cations sont essentiellement transportés sous forme de bicarbonates et accessoirement de sels d'acides faibles. Dans les niveaux supérieurs, une partie importante est certainement, soit solubilisée par des acides organiques, qui, d'après SHILOVA et Korovkina (1965), se trouvent en plus grande quantité à faible profondeur dans les sols podzoliques, soit mise en circulation sous forme de complexes organiques simples.

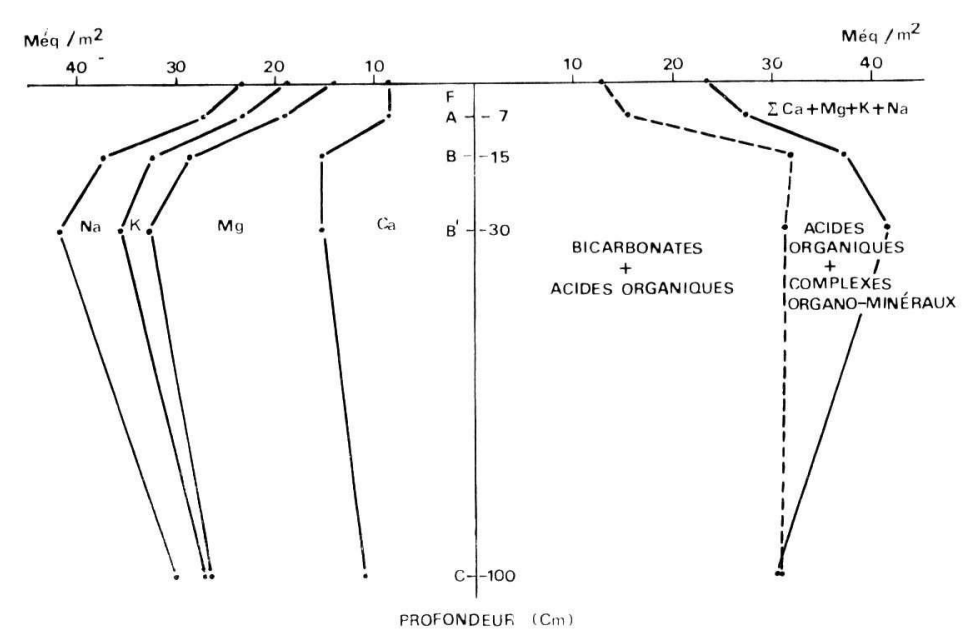

FIG. 4. - Quantité et somme des éléments majeurs dans la solution du sol et somme des bicarbonates (méq $/ \mathrm{m}^{2} / 3$ mois)

FIG. 4. - Amount of cations in the soils solution; total amount of cations and of bicarbonates (méq $\left./ \mathrm{m}^{2} / 3 \mathrm{months}\right)$

Ceci se confirme encore avec le $\mathrm{pH}$ des percolats. Jusqu'à $30 \mathrm{~cm}$, ce dernier est légèrement acide, au-delà il devient basique. Les valeurs moyennes des $\mathrm{pH}$ mesurés sont les suivantes :

$\begin{array}{rr}\text { niveau } & \text { F pH } \\ \text { A } & 6,27 \\ \text { B } & 6,96 \\ \text { B }^{\prime} & 7,01 \\ \text { C } & 7,45\end{array}$

Les échanges et le déplacement des cations dans le sol étudié semblent donc conditionnés par deux interventions distinctes. La première qui comporte essentiellement la solubilisation, dépend à la fois des ions $\mathrm{H}^{+}$libérés par les composés organiques acides, et de l'acide carbonique produit à partir du $\mathrm{CO}_{2} \mathrm{du}$ sol. Le transport des cations ainsi mis en solution se fait sous forme de bicarbonates, de sels d'acides organiques ou de complexes organo-minéraux simples. 
Les observations et les conclusions précédentes sont confirmées en condition artificielle, après arrosage du sol avec de l'eau distillée. Ainsi, l'on constate encore un enrichissement important des percolats en magnésium, suivi du calcium et du sodium (Fig. 5). Comme nous l'avons constaté lors des pluies, le pédochimisme est caractérisé par un déplacement des cations du sol vers la solution dans les 30 premiers centimètres. Au-delà au contraire, l'on assiste à une restitution partielle au complexe absorbant, sauf pour le magnésium. Ce dernier est encore déplacé du sol vers la solution dans les 70 centimètres suivants. Cela a aussi été constaté par GrIER et COLE (1971), au cours d'une expérience d'irrigation dans une clairière située à proximité du placeau étudié dans ce travail. Même quantitativement on retrouve des valeurs similaires à celles obtenues en condition naturelle. Au cours des trois mois d'observation, le sol a bénéficié de $235 \mathrm{~mm}$ de pluie. Les $20 \mathrm{~mm}$ d'eau utilisés expérimentalement représentent une mise en circulation de 12,4 fois moins d'éléments majeurs et de 11,5 fois moins de bicarbonates pour un volume de 12 fois plus petit.

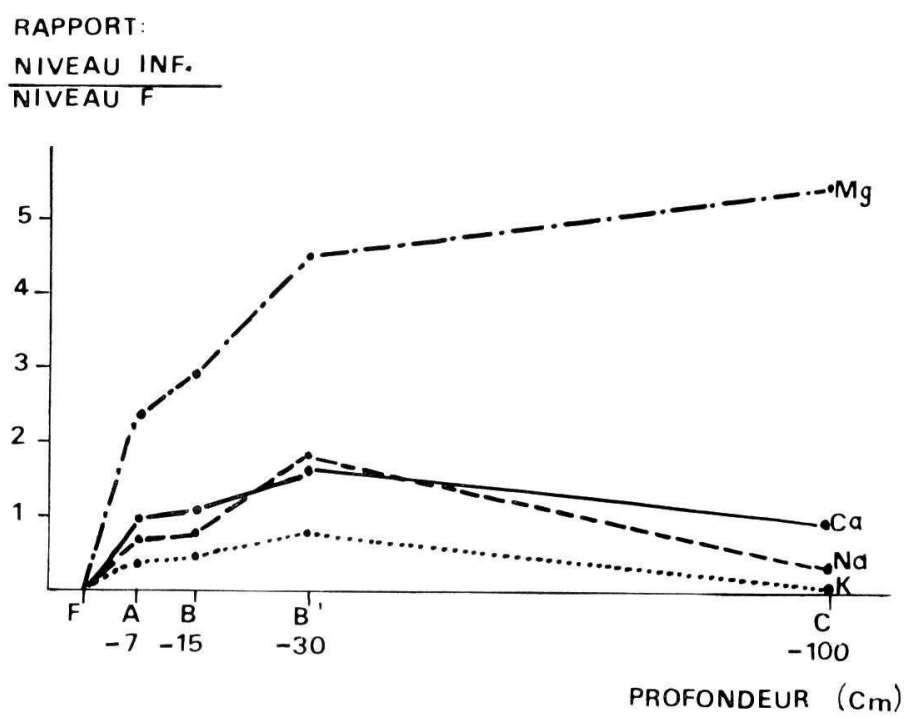

FIG. 5. - Facteur d'augmentation du lessivage des éléments majeurs en fonction de la profondeur du profil traversé par les eaux d'arrosage

FIG. 5. - Coefficient of increase of mineral elements leached at different depths of the soil after an irrigation experiment

L'agent principal de la solubilisation, des échanges et du transport est encore représenté par les anions bicarboniques et à un degré moindre les acides organiques (Fig. 6). Par contre, surtout dans les 15 premiers centimètres du sol, les complexes organiques simples n'interviennent pratiquement pas. Cela résulte certainement de l'absence de produits organiques pluviolessivés, qui par leurs propriétés complexantes contribuent beaucoup au lessivage des horizons supérieurs en condition naturelle. Une preuve en est fournie par les pH moyens des différents percolats. Ceux mesurés en condition artificielle sont moins acides aux niveaux 
F et $\mathrm{A}$, les mêmes qu'en condition naturelle à partir de $15 \mathrm{~cm}$ de profondeur. Les valeurs obtenues sont les suivantes :

$\begin{array}{cr}\text { A } & 6,82 \\ \text { B } & 6,92 \\ \text { B }^{\prime} & 7,00 \\ \text { C } & 7,46\end{array}$

Au-delà de $30 \mathrm{~cm}$ de profondeur, le rôle et les proportions respectives des bicarbonates, des acides organiques et des complexes simples sont les mêmes dans les deux types d'observations.

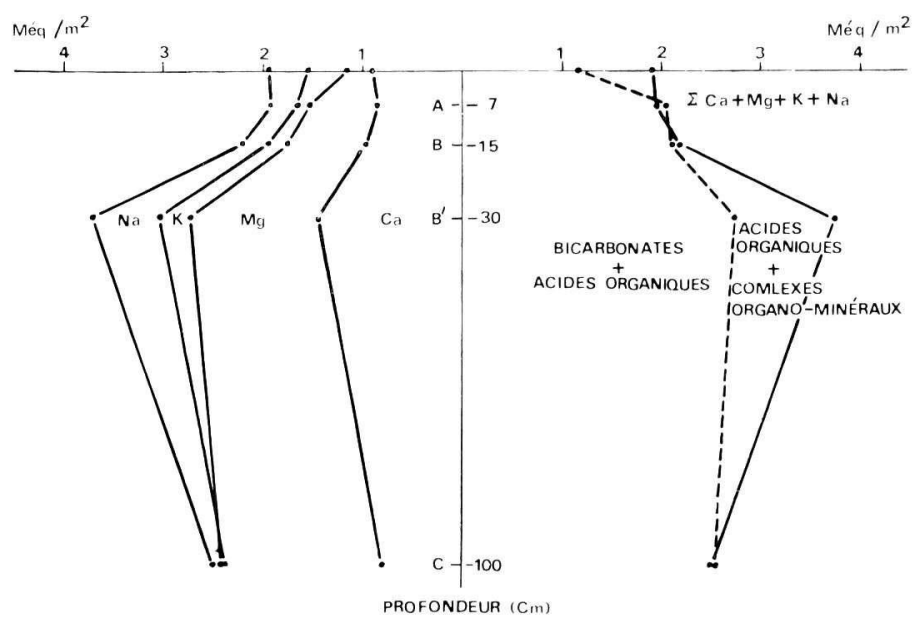

FIG. 6. - Quantité et somme des éléments majeurs dans la solution du sol et somme des bicarbonates après arrosage par $20 \mathrm{~mm}$ d'eau $\left(\mathrm{méq} / \mathrm{m}^{2}\right)$

FIG. 6. - Amount of cations in the soil solution, total amount of cations and of bicarbonates after irrigation with $20 \mathrm{~mm}$ water

L'arrosage permet aussi de suivre l'évolution de la solution du sol au cours du drainage. Dans le cas du pH et des deux cations bivalents (Fig. 7), on constate qu'au début de l'infiltration, la solution est beaucoup plus concentrée, la $\mathrm{pH}$ plus basique, surtout aux niveaux les plus profonds. Puis, peu à peu, la solution lessivant le sol tend à s'acidifier et à se diluer. Seul, le niveau A présente une augmentation de la concentration en calcium avec le temps. Ceci résulte de la mise en solution de cations à partir de la litière en décomposition, ou, au niveau $\mathrm{A}$, par suite d'un apport de produits organiques solubilisés à partir de la même litière. Une fois ces composés entraînés, les échanges ne sont plus dus qu'aux anions bicarboniques, dont la quantité diminue elle aussi avec le temps. Le phénomène est identique pour le $\mathrm{pH}$. Alors que celui des horizons supérieurs devient de moins en moins acide, l'inverse se produit à -15 et $-30 \mathrm{~cm}$. Le dernier fait s'explique par l'effet de la pénétration des lessivats à partir des niveaux supérieurs. Dans ceux-là au contraire, l'entraînement du $\mathrm{CO}_{2}$ et des produits hydrosolubles conduit au résultat inverse. 

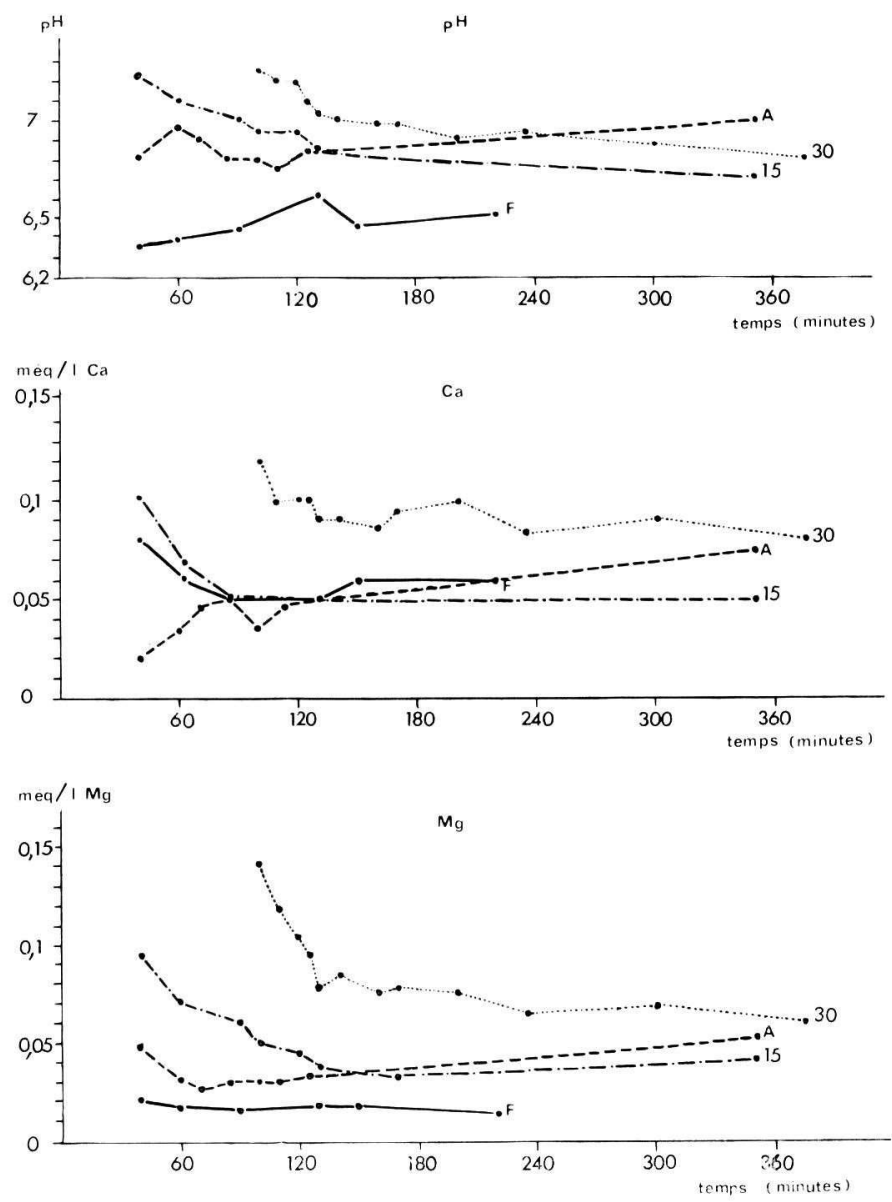

FIG. 7. - Variation du pH et de la teneur en calcium et en magnésium des percolats recueillis à différents niveaux du profil au cours d'un arrosage par $20 \mathrm{~mm}$ d'eau

FIG. 7. - Variation of the $\mathrm{pH}$ and of the calcium and magnesium amount of the leachates collected at different depths, during an irrigation with $20 \mathrm{~mm}$ water

\section{CONCLUSIONS}

L'étude de la composition en éléments majeurs des eaux percolant, après les précipitations, à travers un profil podzolique sous Douglas, permet d'émettre les conclusions suivantes :

1. Les quantités de calcium, magnésium et sodium entraînées augmentent avec la profondeur, alors que celles de potassium diminuent.

2. Dans l'écosystème étudié, en prenant comme point de référence l'horizon F, le lessivage du magnésium est le plus intense. Il est suivi du calcium et du sodium. 
3. Les échanges entre le complexe absorbant et la solution du sol vont du premier vers la solution dans les 30 premiers centimètres, alors que dans les 70 suivants l'on assiste au mouvement inverse, mais en proportion plus faible.

4. Trois agents jouent un rôle dans la solubilisation et le lessivage des cations étudiés. Les anions bicarboniques et les acides organiques, dont l'influence s'étale sur tout le profil; des substances organiques complexantes, dont l'effet est limité en grande partie aux 30 premiers centimètres.

5. Ceci est confirmé par un arrosage du profil, qui montre de plus l'importance des produits pluviolessivés dans la mise en solution des cations.

Reçu pour publication en juin 1973.

\author{
SUMMARY \\ EVOLUTION OF THE CATIONS INVOLVED IN RAIN, CANOPY LEACHATE \\ AND DRAINAGE WATER THROUGH A FOREST ECOSYSTEM
}

The change of $\mathrm{Na}, \mathrm{K}, \mathrm{Ca}$ and $\mathrm{Mg}$ contained in the precipitations after their penetration and transport through the soil is studied in a Douglas Fir forest of the North-West of the United States, by using tension lysimeters.

The balance sheet of the four cations, moving in dissolved phase, was established for the whole ecosystem and the soil subsystem.

The variations of the concentration of the soil solution at different depths was established and some mechanisms are suggested for the solubility and for the transport of cations in this forest soil.

These explanations are confirmed by an irrigation experiment using deionized water.

\title{
ZUSAMMENFASSUNG
}

DYNAMIK DER, IN DEN NIEDERSCHLÄGEN, IN DER KRONENTRAUFE

UND IM BODENSICKERWASSER GELÖSSTEN MINERALELEMENTEN, IN EINEM WALDÖKOSYSTEM

Die Veränderung des $\mathrm{Na}, \mathrm{K}$, Ca und Mg-Gehaltes des Regenwassers welches in den Boden einsickert, wurde in einem Douglas (Pseudotsuga Menziesii Franco) Bestand der Nord-West Küste der U.S.A., mittels Unterdrucklysimetern studiert.

Die Bilanz des Kreislaufes der Fraktion dieser vier Kationen, die sich in Lösung befindet, wurde einerseits für das ganze Ökosystem und anderseits für dessen Boden allein, bestimmt.

Die Veränderung der Konzentration an Bio-Elementen des Bodenwassers, wurde in verschiedenen Tiefen analysiert. Einige Erklärungen über die Solubilisation und den Transport der Kationen sind vorgeschlagen.

Ein Kontrolexperiment, bei künstlicher Bewässerung des Bodens, bestätigt die vorliegenden Erklärungen über den Kationenumsatz im Waldboden.

\section{RÉFÉRENCES BIBLIOGRAPHIQUES}

Bourgeois W. W., LAvkulich L. M., 1972. - A study of forest soils and leachates on sloping topography using a tension lysimeter. Can. J. Soil Sci., 52, 375-391.

Cole D. W., 1968. - A system for measuring conductivity and rate of flow in a forest soil. Water Resour. Res., 4, 1127-1136.

Cole D. W., Ballard T. M., 1968. - Mineral and gaz transfer in a forest floor. A phase model approach. Tree growth and Forest Soils, Youngberg C. I. and Davey C. B. Ed., Corvallis, Oregon, 347-358. 
Cole D. W., Gessel S. P., 1968. - Cedar River Research - a program for studying pathways, rates and processes of elemental cycling in a forest ecosystem. Forest Resour. Monogr. Contrib. $\mathrm{n}^{\circ}{ }^{10}$, University of Washington, Seattle, $53 \mathrm{p}$.

Cole D. W., Gessel S. P., Dice S. F., 1967. - Distribution and cycling of nitrogen, phosphorus, potassium and calcium in a second-growth Douglas-fir ecosystem. Symp. Primary Productivity and Mineral Cycling in Natural Ecosystems. Ecol. Soc. Amer. AAAS Annual Meeting, 197-232.

Duchaufour Ph., 1968. - L'évolution des sols, essai sur la dynamique des profils. Masson et Cie Éd., Paris, 94 p.

Gessel S. P., Cole D. W., 1965. - Influence of removal of forest cover on movement of water and associated elements through soil. J.A.W.W.A., 57, $\mathrm{n}^{\circ} 10,1301-1310$.

Grier C. C., Cole D. W., 1971. - Influence of slash burning on ion transport in a forest soil. Northwest Sci., 45, $\mathrm{n}^{\circ}$ 2, 100-106.

Juste Chr., 1965. - Contribution à la dynamique de l'aluminium dans les sols acides du sud-ouest atlantique; application à leur mise en valeur. Thèse Fac. Sci. Nancy, $143 \mathrm{p}$.

Kilbertus G., 1970. - Étude écologique de la strate muscinale dans une pinède sur calcaire lusitanien en Lorraine. Thèse Fac. Sci. Nancy, 152 p. (ronéo).

Kinderis Z. B., 1970. - Leaching of nutrients by drainage waters. Pochvovedeniye, 2, 102-110.

Mc Coll J. G., Cole D. W., 1968. - A mechanism of cation transport in a temperate forest soil. Northwest Sci., 42, no 4, 134-140.

Rainwater F. H., Thatcher L. L., 1960. - Methods for collection and analyses of water samples. U.S. Geol. Surv. Water Supply Paper 1454, 301 p.

RaPP M., 1971. - Cycle de la matière organique et des éléments minéraux dans quelques écosystèmes méditerranéens. P.B.I. - R.C.P. 40 du C.N.K.S., Éditions du C.N.R.S., Paris, 184 p.

Shilova E. I., Korovkina L. V., 1965. - Composition and properties of podzolic soil solution in a green moss fir-wood based on lysimeter results. Acad. Sci. U.S.S.R., Soil Science, Special Copy (traduit du russe). 